\title{
Conventional Radiographic Assessment of Temporo- mandibular Joint Disorders in Young Saudi Patients: A Retrospective and Prospective Radiographic Study
}

Sultan Mohammed Kaleem, Asif Sheik, Muhammad Ajmal, Muhammad Shahul Hameed, Master Luqman

\begin{abstract}
Objective: The objective of the study is to evaluate temporomandibular joint (TMJ) disorders in young Saudi patients in Southern Aseer Region of the province and to find out the diagnostic efficacy of conventional radiography as basic diagnostic tool.
\end{abstract}

Materials and methods: A random sample of 200 patient radiographic data was collected in which 60 radiographs showing osseous changes are taken as study group. The patients in this study group are then recalled for clinical correlation of the radiographic findings along with the assessment of clinical signs and symptoms, sticking on to the research diagnostic criteria.

Results: There was no statistical difference in age groups and gender of the population and 14 patients $(21.87 \%)$ showed clinical symptoms like clicking and pain in the preauricular region, correlating to the radiographic findings. mostly young female patients reported to have TMJ related symptoms $(29.68 \%)$ whose radiographic findings correlate with that of clinical symptoms.

Conclusion: According to our knowledge, highest prevalence rate of TMDs is found in young Saudi females and is around $43 \%$ and $35 \%$ in young Saudi males. The sensitivity of conventional radiographs in diagnosing TMDs is $78.12 \%$ and specificity is $21.8 \%$.

Keywords: Temporomandibular joint disorders, Conventional radiographs, Anterior disk displacement, Glenoid fossa, Joint space.

How to cite this article: Kaleem SM, Sheik A, Ajmal M, Hameed MS, Luqman M. Conventional Radiographic Assessment of Temporomandibular Joint Disorders in Young Saudi Patients: A Retrospective and Prospective Radiographic Study. Int J Experiment Dent Sci 2013;2(2):76-81.

Source of support: Nil

Conflict of interest: None declared

\section{INTRODUCTION}

The routine dental examination in many undergraduate institutions usually aims to train the student in diagnosing common dental problems and neglect those related to temporomandibular (TMJ) because of the universal agreement among those who treat dysfunction of the TMJ is not possible in undergraduate level and there is a lot of confusion and controversy reign over every aspect of this subject. The key to the successful study of TMJ depends on understanding its unique characteristics which invariably has led to confusion among dentists regarding TMJ dysfunction and treatment. ${ }^{1}$ This discord includes not only diagnosis and treatment but even the definition of TMJ dysfunction.

The aim of this study is to evaluate temporomandibular joint disorders in young Saudi patients reporting to the Outpatient Department in College of Dentistry with existing records and to find out the diagnostic efficacy of conventional radiography as basic diagnostic tool and also to rule out temparomandibular disorder (TMD) patients based on clinical criterion and gender to evaluate them by conventional gold standards. This study is brought about as an eye-opener and guide to the trainees as well as other faculty. Despite years of extensive basic and clinical research, clinicians continue to encounter considerable difficulty in the management of many of these TMJ related conditions. To a large extent, this is related to the lack of accurate diagnosis. ${ }^{1}$

The term TMJ disorder embraces a number of clinical problems that involve the masticatory musculature, the TMJ and associated structure or both. ${ }^{2}$ During the last two decades, there has been dramatic improvement in potential of diagnostic imaging that has lead to significant increase in our understanding of TMJ dysfunction. TMDs cannot be diagnosed on basis of findings by clinical examinations alone, various imaging modalities are very important. ${ }^{2}$ There are four basic questions that should be answered in order to understand the reliability and validity of imaging diagnosis of TMJ disorders, namely:

How accurate are imaging findings? How consistent is the image when several images of the same morphology are obtained over time? How consistent is the interpretation of the images by one observer at several occasions as well as among several different observers? How relevant to the patient's symptomatology are the imaging findings? In addition, cost-effectiveness should be considered for each of the diagnostic test. Imaging should be the prime diagnostic method and considered the most cost-effective way to generate information about the status of the joint. There are multitudes of imaging techniques available, ranging from plain-film to computed tomography (CT) and magnetic resonance (MR) imaging. ${ }^{2}$ This research is concentrated on those techniques that have been most frequently used, such as conventional plain film radiographic procedure (transcranial projection) and digital Pan Eye 
tomography as they are the basic and gold standard techniques for investigating TMJ. Transcranial plain films were used extensively in the past to determine the position of the mandibular condyle within the glenoid fossa and to evaluate osseous changes. ${ }^{3}$ The transcranial film is probably the most commonly used imaging method for study of the TMJ, and this techniques might have some value as a screening modality and for the purpose of documentation, follow-up and patient education. Investigators have suggested that the transcranial projection is reliable for depicting the true morphologic relationship between the condyle and the glenoid fossa. ${ }^{3}$

\section{MATERIALS AND METHODS}

This is an in vivo retrospective and prospective clinical study based on TMJ signs and symptoms in young Saudi population of aseer region of the province. The clinical inclusion criteria for study group were assessed on following five clinical characteristics which were/are modification of those originally described by Okeson. ${ }^{4}$ Study group consists patients with age groups of 20 to 40 years whose radiographs have definitive radiologic findings related to TMJ complex patients with definitive signs and symptoms, like clicking/ popping/Crepitus in the TMJ complex, pain over the preauricular region, early morning stiffness of joint, restricted mouth opening and deflection of mandible to one side up on opening. The exclusion criteria for the study are radiographs with missing 3rd molars, radiographs with poor image quality, radiographs which suggests any orthodontic treatments and patients with cerebral palsy, cardiac pacemakers, patients with obvious skeletal jaw deformities, patients undergoing orthodontic treatment, patients with the history of traumatic extractions, patients who are uncooperative, pregnant and geriatric patients and ankylosis of joint.

Two-hundred digital radiographic data of patients reporting to the outpatient wing of College of Dentistry, King Khalid University, are randomly collected and sorted out depending on age group of the study. These radiographs are examined for any gross osseous changes in the condyle and TMJ complex sticking to the inclusion criteria. The data with the positive radiographic findings is then cross examined by the interobserver and any variance is noted. The subjects whose radiographs reveal osseous changes form the study group and were recalled for clinical examination. The recalled subjects were examined on conventional dental chair by using basic dental instruments and assisted by bimanual palpation of muscles of mastication; a subjective questionnaire is given to rule out the clinical findings, such as jaw pain, joint noise, locking, restricted mouth opening, deviation and tenderness at the TMJ area. Each patient was subjected to clinical diagnostic process in accord with research diagnostic criteria and pain threshold is measured according to the visual analog scale (VAS). Visual analog scale consists of $10 \mathrm{~cm}$ scale with grading from 0 (no pain) to 10 (pain as bad as could be). ${ }^{5}$ The patient marks the point along the line that best represents his or her pain, and their score is measured from the no pain end of the numeric scale and descriptive rating such as no pain, mild, moderate and severe is recorded. Visual analog scale is used here in our study because it is sensitive to treatment effects and can be incorporated into pain diaries and can be used express pain in women population. ${ }^{4}$ The condition of each joint in the patient was categorized according to following diagnosis: NDD (no disk displacement) with MPDS, ADDWR (anterior disk displacement with reduction), ADDWOR (anterior disk displacement without reduction), TMJ arthritis (epiphyte formation, flattening of condyle, erosions in the joint space) and TMJ dislocation.

After the clinical examination, the subjects were taken for the conventional radiographic procedures, such as digital OPG eye panoramic TMJ projection and transcranial projection, the digital radiographs, are assessed by a skilled interobserver for any gross bony abnormalities in the surface of condyle as well as the glenoid fossa and joint space. Condyle position is noted by one of the three methods described by Pullinger and Hollander. ${ }^{5}$ Here in this study, linear measurement of the subjective closest joint space is used where dual linear measurements were made by each examiner at the subjective narrowest posterior and anterior interarticular joint space locations. ${ }^{5}$ The morphologic TMJ changes occurring in the conventional radiographs are evaluated by the experienced oral diagnostician (inter-observer) and a calibration session was preceded by naked eye inspection for macroscopic changes, such as erosions, deviation in form (flattening and osteophytes) using a magnifying viewer with built in light source and a binary registration scale was used to score 0 which indicate no certain of change and score 1 indicate certain of change. The naked eye inspection served as gold standard for the radiographic examination, any interobserver variation is noted. All radiographic images were evaluated for a set criterion to determine osseous changes in the TMJ. Statistical analysis was performed with sensitivity and specificity tests drawn to compare the efficacy of conventional plain film radiography in diagnosis of TMJ disorders and to correlate the radiography/imaging findings to clinical signs and symptoms. 


\section{RESULTS}

The present study is a retrospective clinical study conducted on 200 patient radiographic records collected from department of oral diagnosis and radiology in which 64 radiographs are with osseous changes suggestive of having a TMJ disorder; this study was conducted in the department of maxillofacial surgery and diagnostic sciences - oral diagnosis and radiology wing, College of Dentistry, King Khalid University, Abha, KSA, in the period of 2011 to 2012.

Out of $200(100 \%)$ radiographs examined, $64(32 \%)$ show definitive osseous changes which signifies temporomandibular disorders (TMD) thus forming the study group. One hundred and thirty six out of 200 radiographs (68\%) show no significant osseous change and thus form the control group (Graph 1).

Mean age of the study group was 25.73 years with standard deviation (SD) 5.02 (Table 1).

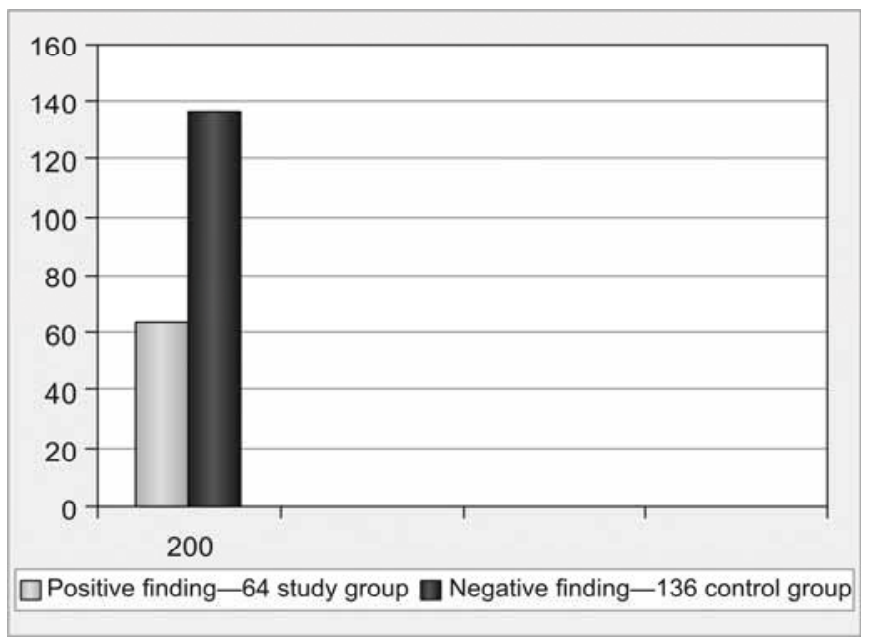

Graph 1: Study and control group distribution

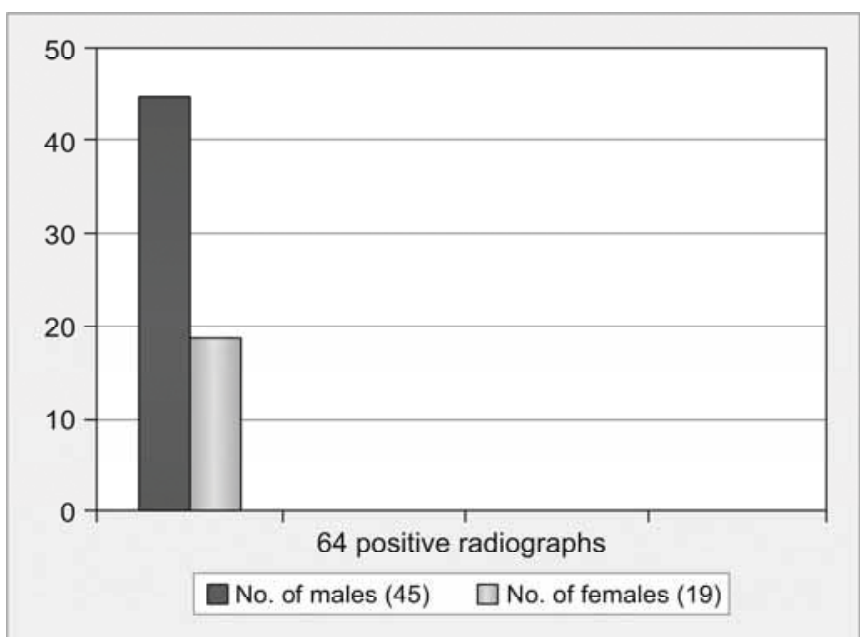

Graph 3: Gender distribution of the study control groups; out of 64 study radiographs, 45 radiographs are of male patients and 19 radiographs are of female, Male: 45 (70.31\%); Female: $19(29.68 \%)$

\section{GENDER DISTRIBUTION OF THE STUDY/CONTROL GROUPS}

Out of 64 study sample, 19 were females $(29.68 \%)$ and 45 were males (70.31\%); in 136 control group, 33 were females (24.26\%) and 103 were males (75.73\%) (Graphs 2 and 3).

\section{SAMPLE DISTRIBUTION WITH RESPECT TO CLINICAL FINDINGS}

\section{Radiographic Significance}

Fourteen $(21.87 \%)$ in the study group of 64 complained of clicking in the joint, preauricular tenderness and stiffness

\begin{tabular}{|c|c|c|}
\hline Gender & Mean age & Std. dev. \\
\hline Male in total 200 radiographs & 37.91 & 4.41 \\
\hline Female in total 200 & 34.63 & 2.39 \\
\hline Mean age in study group of 64 & 25.73 & 5.02 \\
\hline
\end{tabular}

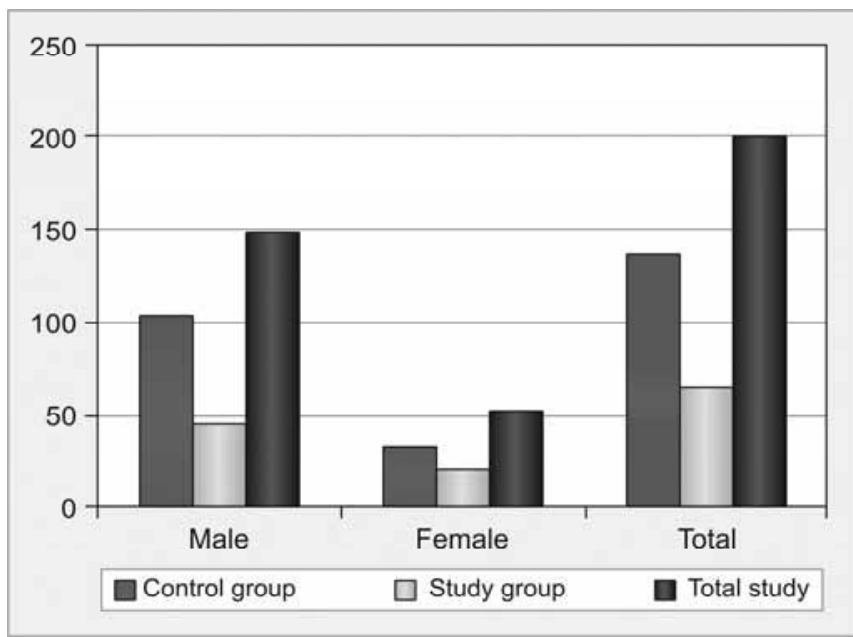

Graph 2: Gender distribution of the study group/control group

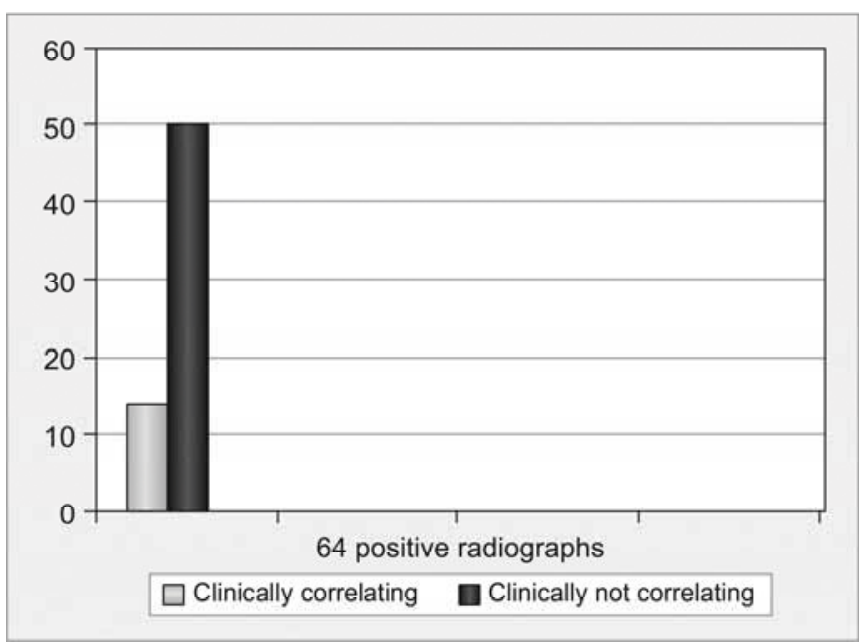

Graph 4: Radiographic and clinical correlation; out of total study group (64): 14 (21.87\%) radiographic findings correlate with clinical symptoms of TMD $50(78.1 \%)$ radiographs did not correlate with clinical symptoms 
in the joint. Fifty $(78.12 \%)$ subjects in the study group has no complaints. The Chi-square test is performed which is equal to 200 with p-value of 0.072 which is less than 0.05 , so, the clinical findings for this study are significant. (Table 2 and Graph 4).

\section{COMPARISON OF STUDY AND CONTROL GROUPS WITH CONVENTIONAL RADIOGRAPHIC PROCEDURE IN DIAGNOSING TMJ DISORDERS}

In 200 radiographs, 64 (32\%) radiographs reveal positive radiographic findings (erosions, flattening, osteophytes and surface irregularities). One hundred and thirty-six radiographs $(68 \%)$ reveal no such findings and hence negative. From 64 study group, $14(21.87 \%)$ produced positive clinical findings suggestive of TMD up on recall. Its chi-square value is equal to 0.72 and its p-value is 0.0935 which is significant at $10 \%$ level of significance (Table 3). Of 14 patients whose

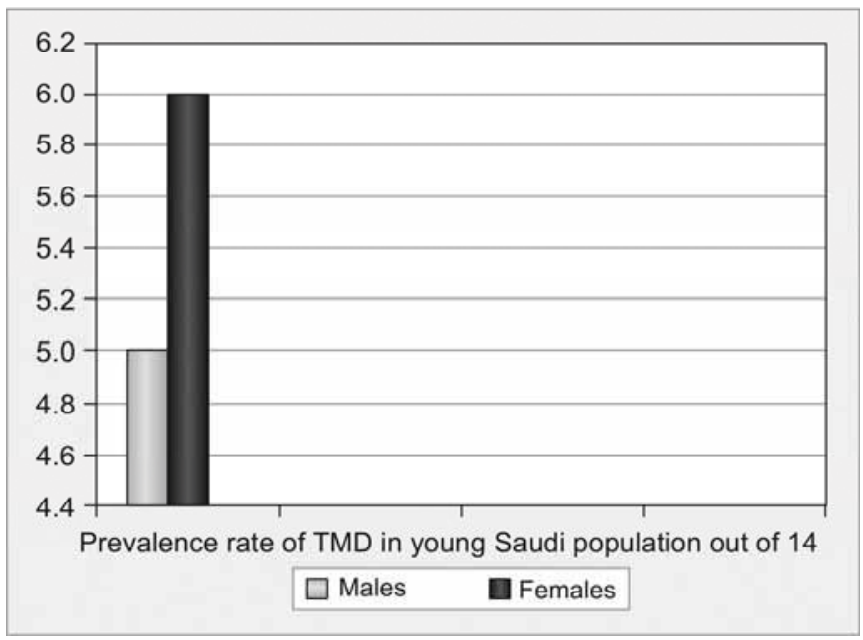

Graph 5: Prevalence of TMD in young Saudi females is around $43 \%$ whose radiographic findings correlate with clinical findings, and it is $35.7 \%$ in males radiographic findings and clinical findings correlate, six patients $(42.8 \%)$ were females and five (35.71\%) were males. Hence, the prevalence of TMDs in male: female is slightly greater in young females compared to young males. positive predictive value (PPV) for conventional radiographs is $21.87 \%$ (Graph 5).

\section{SENSITIVITY AND SPECIFICITY OF CONVENTIONAL RADIOGRAPHS IN DIAGNOSING TMD}

Sensitivity of conventional radiographs in diagnosing TMDs is $78.12 \%$. Specificity of conventional radiographs in diagnosing TMDs is $21.8 \%$ (Graph 6 and Table 4).

\section{DISCUSSION}

Several studies were done to identify clinical signs and symptoms that were predictive of the status of the TMJ.

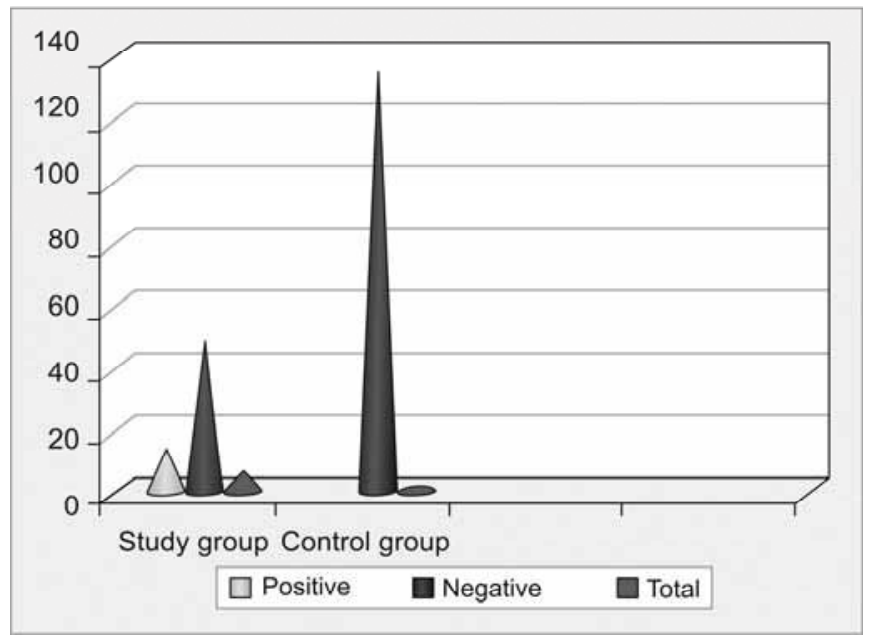

Graph 6: Sensitivity of conventional radiographs in diagnosing TMD's is $78.12 \%$. Specificity of conventional radiographs in diagnosing TMD's is $21.8 \%$

\begin{tabular}{|c|c|c|c|c|c|c|}
\hline Chief complaints & Control & $\%$ & Study & $\%$ & Total & $\%$ \\
\hline Clicking/pain & 0 & 0.00 & 14 & 21.80 & 14 & 7 \\
\hline No & 136 & 68.00 & 50 & 78.12 & 189 & 93 \\
\hline Total & 136 & 100.00 & 64 & 99.92 & 200 & 100 \\
\hline \multicolumn{7}{|c|}{ Chi-square $=0.072^{*}$} \\
\hline
\end{tabular}

*Significant at $5 \%$ level of significance $(p<0.05)$

Table 3: Comparison of study and control groups with conventional radiographic procedure in diagnosing TMJ disorders

\begin{tabular}{lllllll}
$\begin{array}{l}\text { Conventional radiographic } \\
\text { procedure }\end{array}$ & Control & $\%$ & Study & $\%$ & Total & $\%$ \\
Negative & 136 & 68.00 & 50 & 78.12 & 186 & 93 \\
Positive & 0 & 00.00 & 14 & 21.87 & 14 & 7 \\
\hline Total & 136 & 68.00 & 64 & 99.99 & 200 & 100 \\
& \multicolumn{2}{c}{ Chi-square $=0.72, p=0.0935^{* *}$} \\
\end{tabular}

${ }^{* *}$ Significant at $10 \%$ level of significance $(p<0.10)$ 


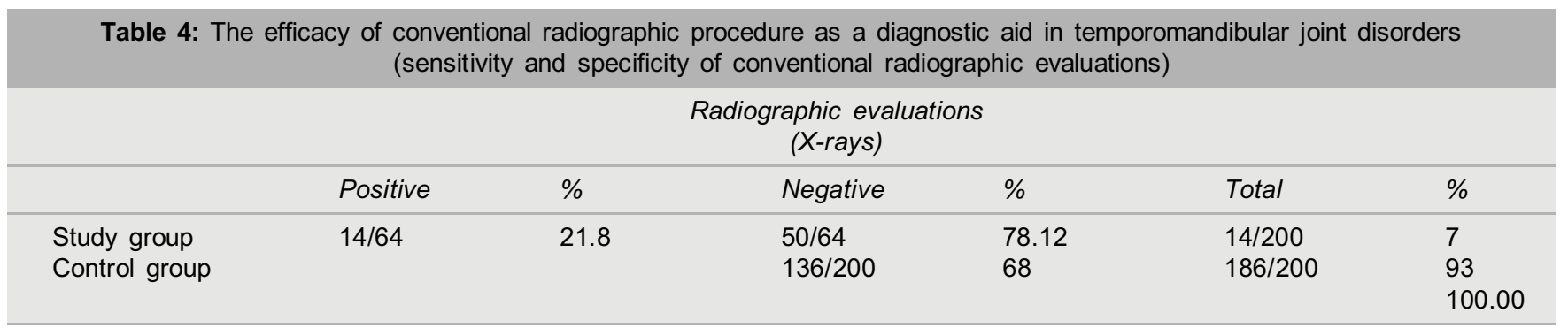

Various diagnostic imaging techniques were performed which contributed to proper diagnosis of TMJ disorders. The commonest and most well-established technique for examining the TMJ is the conventional radiography. Even clearer depictions of the osseous changes were gained from conventional tomography. ${ }^{3}$ In our study, there was uniform agreement between the three observers in ruling out the osseous changes seen in the conventional radiographs. The average interobserver agreement in the study was $95 \%$ and the observers were consistent among themselves as a group. All imaging techniques are not equally effective for each of the different conditions affecting TMJ. The accuracy of clinical examination for diagnosing the exact status of TMD has been investigated in various studies ${ }^{6}$ a morphologic variation without evidence of tissue pathology may actually be a developmental adaptation to any combination of intrinsic and extrinsic factors with the resulting functional equilibrium being the most physiologic relationship for that particular individual. ${ }^{6}$ Computed tomography provides clinicians with the ability to investigate the inner depths of human anatomy slice by slice through computer reformation of the image but the patient radiation exposure is three-fold, when compared to conventional radiography. ${ }^{7}$ In this study, imaging of bony abnormalities is adequately accomplished by conventional radiographic procedures (sensitivity $78.12 \%$ and specificity of $21.8 \%$ ).

The most common clinical sign in our study group was clicking followed preauricular tenderness in the joint (p-value 0.072 ) which is less than $0.05 \%$ significance, which denotes this study to be significant in comparing the imaging findings with that of clinical symptoms of pain. In a study carried out by Knobs $\mathrm{G}$ et al concluded that although clicking is predictor of anterior disk displacement with reduction, it was not present in the entire study population. ${ }^{8}$ In the present study, $21.87 \%$ of subjects produce clinical finding of clicking or popping noise in the TMJ, signs of disease like pain and $78.12 \%$ subjects with no signs and symptoms. The $\mathrm{p}$-value considered in accordance of clinical findings in our study is 0.0933 which is significant in accord with $10 \%$ level of significance. Expressing the accuracy of the imaging modality (conventional plain film radiography, OPG pan eye tomography, transcranial projection) as the number of radiographic scores differing from the gold standards resulted in a single-valued parameter, which provided a greater separation of the modalities than using traditional sensitivity and specificity values. With this definition of accuracy, the present results showed a significantly less diagnostic accuracy using a conventional plain tomography in ruling out bony changes in TMDs. Independent of projections the highest positive predictive value is obtained with transcranial radiographs. With the advent of newer modifications in the conventional radiography and specialized techniques, such as cone beam CT and TACT, the sensitivity and specificity for ruling out changes in the joint are increased four-fold and should be brought into institutional practices for diagnosing and monitoring the treatment in TMJ disorders.

\section{SUMMARY}

The present study consisted of randomly selected 200 patient radiographic data out of which 64 radiographs are with findings suggestive of TMDs these were recalled and subjected to clinical diagnostic procedures in accordance with the RDC/TMD. Clinical diagnosis was made based on clinical signs and symptoms, each individual was subjected for conventional radiographic, specialized TMJ radiographic projections and the radiographs were interpreted by experienced oral and maxillofacial radiologists and a consensus for diagnosis was taken from all the three interpretation parameters and compared with clinical diagnosis. The results were statistically analyzed and sensitivity of $78 \%$ and specificity of $21 \%$ was observed for RDC diagnosis with respect to conventional radiographic procedures in the total sample. The following inference was drawn from the study. The prevalence of TMD is slightly higher in the age groups of 25 years, females are affected more in the ratio 1.5: 1 , clicking and preauricular tenderness are the most common clinical manifestations. Conventional radiography play limited role as an inexpensive screening method either to show degenerative changes or to exclude old traumatic abnormalities or congenital abnormalities that may mimic TMDs which provide better resolution images with minimal radiation exposure. There is considerable difference among the study group in the clinical diagnostic 
criteria which were thought to be the predictors in arriving at the definitive diagnosis, when compared to radiological diagnosis.

\section{CONCLUSION}

The present study depicts conventional radiographic procedures as golden standard among other modalities in ruling out bony alterations caused due to TMDs. And, they are made as a basic preliminary imaging modality in ruling out TMDs. The clinical findings as suggested by RDC, alone were not sufficient for diagnosis of TMJ disorders as it needs further reevaluation and revision with modern imaging techniques, like CBCT, TACT. The imaging modality used in our study is not significant in ruling out the disk position in the TMDs for which other specialized techniques, such as MRI and arthrography, have to be performed for ruling out disk displacements. With the advent of modern imaging techniques, like CT and MRI, both soft and hard tissues can now be viewed in nearly any desired plane of reference with considerable accuracy.

\section{REFERENCES}

1. Pettigrew J, Roberts D. Identification of an anteriorly displaced meniscus in vitro by means of three-dimensional image reconstructions. Oral Surg Oral Med Oral Pathol Oral Radiol Endod 1985;59:535-542.

2. Westesson PL. Reliability and validity of imaging diagnosis of temporomandibular joint. Adv Dent Res 1993;7(2):137-151.

3. Katzberg RW. Temporomandibular joint imaging. J Radiology 1989;170:297-307.

4. Blasberg B, Greenberg MS. Burkett's tenth edition. Temporomandibular Disorders, p 271-299, 310.
5. Pullienger A, Hollander L. Assessment of mandibular condylar position: a comparison of transcranial radiographs and linear tomograms. Oral Surg Oral Med Oral Pathol Oral Radiol Endod 1985;60:329-334.

6. Katzberg RW, Keith D. Internal derangements of TMJ: an assessment of condylar position in centric occlusion. J Prosthet Dent 1983;49(2):250-253.

7. Roberts $\mathrm{C}$, et al. The clinical predictability of internal derangements of temporomandibular joint. Oral Surg Oral Med Oral Pathol Oral Radiol Endod 1991;71:412-414.

8. Helms CA. Diagnostic imaging of TMJ: recommendations for use of various techniques. AJR 1990;154:319-322.

\section{ABOUT THE AUTHORS}

\section{Sultan Mohammed Kaleem (Corresponding Author)}

Assistant Professor, College of Dentistry, King Khalid University Abha-Asir Province, Saudi Arabia, e-mail: kaleemds@rediffmail.com

\section{Asif Sheik}

Assistant Professor, College of Dentistry, King Khalid University Abha-Asir Province, Saudi Arabia

\section{Muhammad Ajmal}

Assistant Professor, College of Dentistry, King Khalid University Abha-Asir Province, Saudi Arabia

\section{Muhammad Shahul Hameed}

Assistant Professor, College of Dentistry, King Khalid University Abha-Asir Province, Saudi Arabia

\section{Master Luqman}

Assistant Professor, College of Dentistry, King Khalid University Abha-Asir Province, Saudi Arabia 